Table. Spearman Correlations between CRISS and individual components at 12 months and Comparison of ABA and PBO using CRISS index and individual components at 12 months;

\begin{tabular}{|c|c|c|c|c|c|}
\hline Outcome & & $\begin{array}{l}\mathrm{ABA} \\
\mathrm{N}=44\end{array}$ & $\begin{array}{l}\mathrm{PBO} \\
\mathrm{N}=44\end{array}$ & $\begin{array}{c}\text { Treatment } \\
\text { Difference (ABA- } \\
\text { PBO) }\end{array}$ & $\begin{array}{c}\mathrm{P}- \\
\text { value }^{\wedge}\end{array}$ \\
\hline \multirow[t]{2}{*}{$\begin{array}{l}\text { ACR CRISS (0.0-1.0) } \\
\text { median (IQR) }\end{array}$} & & $\begin{array}{c}0.68 \\
(1.00)\end{array}$ & $\begin{array}{c}0.01 \\
(0.86)\end{array}$ & & 0.03 \\
\hline & $\begin{array}{l}\text { Spearman } \\
\text { Correlation }\end{array}$ & $\begin{array}{l}\text { LS } \\
\text { mean } \\
(\mathrm{SE})\end{array}$ & $\begin{array}{l}\text { LS } \\
\text { mean } \\
(\mathrm{SE})\end{array}$ & $\begin{array}{l}\text { LS mean } \\
\quad(\mathrm{SE})\end{array}$ & $\begin{array}{c}\mathrm{P}- \\
\text { value }^{\wedge \wedge}\end{array}$ \\
\hline$\Delta \mathrm{mRSS}(0-51)$ & $-0.75^{\star}$ & $\begin{array}{c}-6.7 \\
(1.30)\end{array}$ & $\begin{array}{c}-3.8 \\
(1.23)\end{array}$ & $-2.9(1.75)$ & 0.10 \\
\hline$\triangle \mathrm{FVC} \%$ predicted & $0.36^{*}$ & $\begin{array}{c}-1.4 \\
(1.30)\end{array}$ & $\begin{array}{c}-3.1 \\
(1.20)\end{array}$ & $1.7(1.72)$ & 0.32 \\
\hline$\triangle$ PTGA $(0-10)$ & -0.17 & $\begin{array}{c}-0.50 \\
(0.392)\end{array}$ & $\begin{array}{l}-0.30 \\
(0.385)\end{array}$ & $-0.20(0.557)$ & 0.73 \\
\hline$\triangle \mathrm{MDGA}(0-10)$ & $-0.47^{*}$ & $\begin{array}{c}-1.34 \\
(0.282)\end{array}$ & $\begin{array}{l}-0.18 \\
(0.284)\end{array}$ & $-1.16(0.403)$ & 0.004 \\
\hline$\triangle \mathrm{HAQ}-\mathrm{DI}(0-3)$ & -0.21 & $\begin{array}{c}-0.11 \\
(0.079)\end{array}$ & $\begin{array}{c}0.11 \\
(0.076)\end{array}$ & $-0.22(0.108)$ & 0.05 \\
\hline
\end{tabular}

${ }^{\wedge} \mathrm{p}$-value for treatment comparisons based on Van Elteren test ${ }^{\wedge} \mathrm{p}$-value for treatment comparisons based on ANCOVA model with treatment, duration of SSc and baseline value as covariates ${ }^{*} p<0.01$ using Spearman correlation coefficientNegative score denotes improvement, except for FVC\% where negative score denotes worsening; LS mean = least squares mean; $\mathrm{SE}=$ standard error

\section{FRI0328 BRANCHED CHAIN AMINO ACIDSIN THE TREATMENT OF POLYMYOSITIS AND DERMATOMYOSITIS: RESULTS FROM THE BTOUGH STUDY}

Naoki Kimura, Hitoshi Kohsaka. Tokyo Medical and Dental University (TMDU), Department of Rheumatology, Tokyo, Japan

Background: Muscle functions of patients with polymyositis and dermatomyositis (PM/DM) remain often impaired even after successful control of the immune-mediated muscle injury by immunosuppressive therapy. The only effort at the present to regain muscle functions except for the immunosuppression is rehabilitation, which is carried out systematically in limited institutes. No medicines for rebuilding muscles have been approved. Branched chain amino acids (BCAA) promote skeletal muscle protein synthesis and inhibit muscle atrophy. They thus have positive effects on muscle power, but have never been examined for the effects on PM/DM patients.

Objectives: To assess the efficacy and safety of BCAA in the treatment of PM/DM for official approval of their use in Japan.

Methods: Untreated adults with PM/DM were enrolled in a randomized, double-blind trial to receive either TK-98 (drug name of BCAA) or placebo in addition to the conventional immunosuppressive agents. One package of TK-98 $(4.15 \mathrm{~g})$ contained L-isoleucine 952mg, L-leucine $1904 \mathrm{mg}$, and L-valine $1144 \mathrm{mg}$ (molar ratio is 1:2:1.35), and 6 packages were administered daily in 3 divided doses. After 12 weeks, patients with average manual muscle test (MMT) score less than 9.5 were enrolled in an open label extension study for 12 weeks. The primary end point was the change of the MMT score at 12 weeks. The secondary end points were the disease activity evaluated with myositis disease activity core set (MDACS) and the change of functional index (FI), which evaluates dynamic repetitive muscle functions.

Results: Forty-seven patients were randomized to the TK-98 (24 patients [12 with PM and 12 with DM]) and placebo (23 patients [11 with PM and 12 with DM]) groups. The baseline MMT scores were equivalent $(7.97 \pm 0.92$ [mean $\pm S D$ ] in the TK-98 group and $7.84 \pm 0.86$ in the placebo group). The change of MMT scores at 12 weeks were $0.70 \pm 0.19$ (mean \pm SEM) and $0.69 \pm 0.18$, respectively $(P=0.98)$. Thirteen patients from the TK-98 group and 12 from the placebo group were enrolled in the extension study. The MMT scores in both groups improved comparably throughout the extension study. The increase of the $\mathrm{FI}$ scores of the shoulder flexion at 12 weeks was significantly larger in the TK-98 group $(27.9 \pm 5.67$ and $12.8 \pm 5.67$ in the right shoulder flexion $[P<0.05], 27.0$ \pm 5.44 and $13.4 \pm 5.95$ in the left shoulder flexion $[P<0.05]$ ). The improvement rate of the average $\mathrm{FI}$ scores of all tested motions (head lift, shoulder flexion, and hip flexion) through the first 12 weeks was larger in the TK-98 group. No difference was found in the disease activity throughout the study period. Frequencies of the adverse events until 12 weeks were comparable.
Conclusion: Although BCAA exerted no effects in the improvement of the muscle strength evaluated with MMT, they were effective in the improvement of dynamic repetitive muscle functions in patients with PM/DM with out significant increase of adverse events.

Disclosure of Interests: None declared

DOI: 10.1136/annrheumdis-2019-eular.5235

\section{FRI0329 ANALYSIS OF 11 CASES OF ANTI-PL-7 ANTIBODY POSITIVE PATIENTS WITH IDIOPATHIC INFLAMMATORY MYOPATHIES. MALIGNANCY MAY NOT BE UNCOMMON COMPLICATION IN ANTI-PL-7 ANTIBODY POSITIVE MYOSITIS PATIENTS}

Taiga Kuga, Yoshiyuki Abe, Masakazu Matsushita, Kurisu Tada, Ken Yamaji, Naoto Tamura. Juntendo University School of Medicine, Department of Internal Medicine and Rheumatology, Tokyo, Japan

Background: Various autoantibodies are known to be related to idiopathic inflammatory myopathies (IIM). Anti-PL-7 antibody is anti-threonyl-tRNA synthetase antibody associated with antisynthetase syndrome (ASS). Since anti-PL-7 antibody is rare (mostly $1-4 \%$ of myositis, while a Japanese study reported 17\%), little is known as to clinical characteristics of it (1). Objectives: To analyze clinical characteristics of anti-PL-7 positive IIM patients.

Methods: Anti-PL-7 antibody was detected by EUROLINE Myositis Profile 3. IIM diagnosis was made by the 2017 EULAR/ACR classification criteria (2) and/or Bohan And Peter classification (3). Eleven anti-PL-7 antibody positive adult patients (all female), age at onset (61.5 \pm 12.6 years) were enrolled in this study between 2009 and 2018. Clinical manifestations, laboratory and instrumental data were reviewed in this single centre retrospective cohort.

Results: Characteristic symptoms were identified at diagnosis: skin manifestations (7/11 cases, $63.6 \%)$, muscle weakness (8/11 cases, $72.7 \%)$, arthralgia (5/11 cases, 45.5\%) and Raynaud's phenomenon (4/11 cases $36.4 \%$ ). Myogenic enzymes were elevated in most cases (10/11 cases, $90.9 \%$ ). ILD was detected in all patients (11/11 cases, $100 \%$ ) and 2 patients (18.2\%) developed rapidly progressive ILD. Largest IIM subtype was polymyositis (PM, 5/11 cases), followed by dermatomyositis (DM, 3/ 11 cases) and amyopathic dermatomyositis (ADM, 3/11 cases). Five patients $(45.5 \%)$ complicated with malignancy within 3 years from the diagnosis of IIM. Though clinical manifestations and laboratory data showed any difference between malignancy group and non-malignancy group, all 3 ADM cases but no DM cases complicated with malignancy in this study.

Conclusion: Anti-PL-7 antibody positive IIM patients frequently complicated with ILD. Frequency of cancer in ASS patients within three years from diagnosis was $1.7 \%$ and not much different from the general population in previous report from France (4). Though this study only included IIM patients and may have selection bias, careful malignancy survey may be essential in Anti-PL-7 antibody positive IIM patients.

\section{REFERENCES}

[1] Y Yamazaki, et al. Unusually High Frequency of Autoantibodies to PL-7 Associated With Milder Muscle Disease in Japanese Patients With Polymyositis/DermatomyositisARTHRITIS \& RHEUMATISM Vol. 54, No. 6 , June 2006, pp 2004-2009

[2] Lundberg IE, Tjärnlund $A$, Bottai $M$, et al. EULAR/ACR classification criteria for adult and juvenile idiopathic inflammatory myopathies and their Major Subgroups. Ann Rheum Dis. 2017;76:1955-64.

[3] Bohan A, Peter J. Polymyositis and dermatomyositis. N Engl J Med 1975, 292: 344-347; 403-407.

[4] Hervier B, et al. Hierarchical cluster and survival analyses of antisynthetase syndrome: phenotype and outcome are correlated with anti-tRNA synthetase antibody specificity. Autoimmunity reviews. 2012; 12:210-217.

Disclosure of Interests: Taiga Kuga: None declared, Yoshiyuki Abe: None declared, Masakazu Matsushita: None declared, Kurisu Tada Grant research support from: Eli Lilly, Ken Yamaji: None declared, Naoto Tamura Grant/research support from: Astellas Pharma Inc., Asahi Kasei Pharma, AYUMI Pharmaceutical Co., Chugai Pharmaceutical Co. LTD, Eisai Inc., :Takeda Pharmaceutical Company Ltd., Speakers bureau: Jans sen Pharmaceutical K.K., Bristol-Myers Squibb K.K., :Mitsubishi Tanabe Pharma Co.

DOI: 10.1136/annrheumdis-2019-eular.4150 